Draft Version August 3, 2021

Typeset using LATEX twocolumn style in AASTeX61

\title{
APPROXIMATING PHOTO-Z PDFS FOR LARGE SURVEYS
}

\author{
A.I. Malz,${ }^{1,2}$ P.J. Marshall,${ }^{3,4}$ J. DeRose, ${ }^{4,5}$ M.L. Graham, ${ }^{6}$ S.J. Schmidt ${ }^{7}$ and R. WechsleR ${ }^{4}$ \\ (LSST DARK ENERGy SCIENCE COLlaboration)
}

\author{
${ }^{1}$ Center for Cosmology and Particle Physics, New York University, 726 Broadway, New York, 10003, USA \\ 2 Department of Physics, New York University, 726 Broadway, New York, 10003, USA \\ ${ }^{3}$ SLAC National Accelerator Laboratory, Menlo Park, CA 94025, USA \\ ${ }^{4}$ Kavli Institute for Particle Astrophysics 83 Cosmology, P. O. Box 2450, Stanford University, Stanford, CA 94305, USA \\ ${ }^{5}$ Department of Physics, Stanford University, 382 Via Pueblo Mall, Stanford, CA 94305, USA \\ ${ }^{6}$ Department of Astronomy, University of Washington, Box 351580 U.W., Seattle, WA 98195-1580, USA \\ ${ }^{7}$ Dept. of Physics, University of California, One Shields Ave., Davis, CA, 95616, USA
}

\begin{abstract}
Modern galaxy surveys produce redshift probability density functions (PDFs) in addition to traditional photometric redshift (photo- $z$ ) point estimates. However, the storage of photo- $z$ PDFs may present a challenge with increasingly large catalogs, as we face a trade-off between the accuracy of subsequent science measurements and the limitation of finite storage resources. This paper presents qp, a Python package for manipulating parametrizations of 1-dimensional PDFs, as suitable for photo- $z$ PDF compression. We use qp to investigate the performance of three simple PDF storage formats (quantiles, samples, and step functions) as a function of the number of stored parameters on two realistic mock datasets, representative of upcoming surveys with different data qualities. We propose some best practices for choosing a photo- $z$ PDF approximation scheme and demonstrate the approach on a science case using performance metrics on both ensembles of individual photo- $z$ PDFs and an estimator of the overall redshift distribution function. We show that both the properties of the set of PDFs we wish to approximate and the fidelity metric(s) chosen affect the optimal parametrization. Additionally, we find that quantiles and samples outperform step functions, and we encourage further consideration of these formats for PDF approximation.
\end{abstract}

Keywords: methods: statistical, methods: miscellaneous, astronomical databases: miscellaneous, catalogs, galaxies: distances and redshifts

\section{INTRODUCTION}

Upcoming wide-field imaging surveys such as Large Synoptic Survey Telescope (LSST) ${ }^{1}$ (Ivezić et al. 2008) will observe tens of billions of galaxies photometrically, without follow-up spectroscopy. Over the past decade, the Kilo-Degree Survey ${ }^{2}$, Hyper Suprime-Cam Subaru Strategic Program ${ }^{3}$, and Dark Energy Survey ${ }^{4}$ have paved the way for LSST via similar survey strategies on tens of millions of galaxies. Studies of precision cosmology and galaxy evolution with the anticipated data will thus rely almost exclusively on the method of photometric redshift (photo- $z$ ) estimation. Photo- $z$ s are subject to a number of systematic errors, some caused by the estimation procedures and others intrinsic to the

\footnotetext{
1 https://www.lsst.org/

2 http://kids.strw.leidenuniv.nl/

3 http://hsc.mtk.nao.ac.jp/ssp/

4 https://www. darkenergysurvey.org/
}

data itself. For the purpose of producing public photo$z$ catalogs, the redshift estimation community has thus come to favor methods that provide a photo- $z$ probability density function (PDF) conveying the potential for such systematic errors for each galaxy in the survey (Tanaka et al. 2018; de Jong et al. 2017; Sheldon et al. 2012).

Given that the photo- $z$ PDF catalogs of ongoing surveys already include $\sim 10^{7}$ galaxies, and that those of upcoming surveys will include $\sim 10^{10}$ galaxies, storage of these PDFs must balance accuracy of the catalog against limited storage resources. For example, the LSST's anticipated public catalog will be limited to $\sim 100$ floating point numbers per galaxy for all information characterizing the redshift (Juric et al. 2017, Section 4.2.2), including photo- $z$ PDFs derived by multiple methods. Furthermore, the problem of storing PDFs 
is not unique to galaxy surveys. $\mathrm{Gaia}^{5}$, for example, has committed to providing a catalog of PDFs of stellar properties including velocities, so an approach to optimizing the choice of PDF storage parametrization could benefit astronomy more broadly.

Carrasco Kind \& Brunner (2014b) first addressed the question of approximating photo- $z$ PDFs in the context of a particular galaxy survey and metrics applicable to deterministic, not probabilistic, data products. However, we expect the optimal choice of photo- $z$ PDF storage approximation to depend on the intended science applications and their requirements on photo- $z$ PDF accuracy as well as the properties of the anticipated photo- $z$ PDFs. Different science cases will need different metrics, and different formats may be appropriate for different datasets. In this paper, we address the question of how these choices should be made, by providing the publicly available qp Python package ${ }^{6}$ (Malz \& Marshall 2017) enabling each survey to optimize their photo- $z$ PDF approximation via mathematically motivated and sciencedriven metrics. We demonstrate this approach on two sets of realistic mock data in the context of LSST.

In Section 2, we outline how qp can be used to optimize the choice of photo- $z$ PDF parametrization. In Section 3, we describe the mock datasets on which we demonstrate such an analysis. We present the results of this procedure in Section 4 and make recommendations for the use of qp by the photo- $z$ community in Section 5 .

\section{METHODS}

We have developed the qp Python package to facilitate the approximation of one-dimensional PDFs, including photo- $z$ PDFs, and comparisons between approximations.

A qp.PDF object is associated with sets of parameters for each approximation considered. Conversions between approximations are facilitated by the numpy ${ }^{7}$ (Walt et al. 2011), scipy ${ }^{8}$ (Jones et al. 2001), and scikit-learn ${ }^{9}$ (Pedregosa et al. 2011) tools. The currently supported parametrizations are described in Section 2.1 .

The qp package also provides a few built-in metrics for the accuracy of a representation of a PDF relative to a given reference representation. Built-in plots are

\footnotetext{
${ }^{5}$ https://www.gaia-eso.eu/

6 https://github.com/aimalz/qp

7 http://www. numpy.org/

8 https://www.scipy.org/

${ }^{9}$ http://scikit-learn.org
}

made using matplotlib ${ }^{10}$ (Hunter 2007). A subset of the included metrics is described in Section 2.2.

Catalog-level manipulations are performed using the qp. Ensemble class that serves as a wrapper for operations over collections of qp.PDF objects. Parallelization is facilitated by the pathos ${ }^{11}$ (McKerns et al. 2012; McKerns \& Aivazis 2010) package.

\subsection{Approximation Methods}

First, we establish a vocabulary for the approximations. Each parametrization of a photo- $z$ PDF is defined in terms of the parameters $\vec{c}$ unique to its galaxy, the metaparameters $\vec{C}$ shared over many galaxies, and the format function $\mathcal{F}$ that reconstructs a PDF from its parameters and metaparameters. A parametrization in turn corresponds to a representation

$$
\hat{p}^{\mathcal{F}, \vec{C}, \vec{c}}(z) \equiv \mathcal{F}_{\vec{C}}(z ; \vec{c})
$$

of the approximated photo- $z \mathrm{PDF}$, denoted as $\hat{p}(z)$ for brevity. The dimensionality of $\vec{c}$ is the number $N_{f}$ of stored parameters per photo- $z$ PDF, which are presumed to be scalar numbers unless otherwise specified. The number of elements of $\vec{C}$ is of little significance so long as the metaparameters do not have storage requirements that are competitive with those of the ensemble of per-galaxy parameters.

While it is possible to construct a catalog where $N_{f}$ is not shared among all members but is instead optimized for each galaxy, we restrict this study to metaparameters shared over all galaxies considered. A non-uniform $\vec{C}$ scheme would have the advantage of re-allocating storage resources from galaxies whose photo- $z$ PDFs can be fully characterized by very few numbers to galaxies with highly featured photo- $z$ PDFs that require more numbers for complete characterization; consider the case of a delta function photo- $z$ PDF for which no more information is preserved for any elements of $\vec{c}$ than the location of the peak as opposed to the case of a highly multimodal photo- $z$ PDF whose shape cannot be easily parametrized by any known functional form and would benefit from the $N_{f}-1$ parameters wasted on the galaxy with a trivial shape. However, no galaxy survey mission has yet proposed this scheme, so we could thus only speculate as to the potential scope and specific goals of such an optimization procedure. Thus, we postpone such an investigation and eagerly anticipate future consideration of this possibility.

\footnotetext{
10 https://matplotlib.org/

11 http://trac.mystic.cacr.caltech.edu/project/pathos/ wiki.html
} 


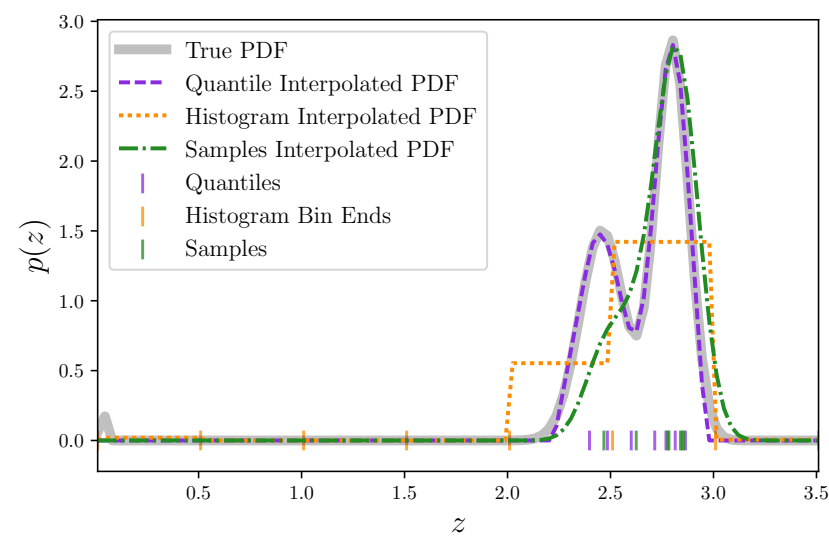

Figure 1. qp approximation of a continuous 1-dimensional PDF (thick, solid gray line) using: the step function (orange dotted line), samples (green dash-dotted line), and quantile formats (purple dashed line) with the same number of stored parameters $\left(N_{f}=7\right.$ in this case).

qp currently supports conversion of photo- $z$ PDF approximations between five formats: step functions, samples, quantiles, evaluations, and functional forms, which may be general mixture models of PDFs from a comprehensive library of those with functional forms implemented as scipy.rv_continuous objects. When the format function is not specified by the approximation, we refer to a generic interpolator function $F_{\vec{C}^{\prime}}\left(z ; \vec{c}, \mathcal{F}_{\vec{C}}\right)$ with its own metaparameters $\vec{C}^{\prime}$, which must be chosen by the researcher. qp supports numerous interpolation schemes, including several from the popular scipy.interpolate library.

In this work we consider special cases of three of these formats as candidates for large survey photo- $z$ PDF catalog storage: regular binning (Section 2.1.1), random samples (Section 2.1.2), and regular quantiles (Section 2.1.3). Step functions and samples have been used in published analyses, and we introduce the quantile format because of its favorable statistical properties. We do not consider the function evaluation format because it is statistically very similar to step functions, and we exclude at this time the mixture model format because it is only appropriate when the underlying PDFs are actually mixture models, which we do not guarantee in this study. The three formats we investigate are illustrated on a multimodal PDF in Figure 1.

In spite of its impressive compression properties, we have not yet included the SparsePz ${ }^{12}$ sparse basis representation of Carrasco Kind \& Brunner (2014b), in which the parameters are the integer identifiers of $N_{f}$ mixture

12 https://github.com/mgckind/SparsePz model components from a library of $\sim 10^{4}$ functions. We omit this format because decomposition with SparsePZ does not enforce the condition that the representation be a PDF in the mathematical sense of nonnegativity and integration to unity. While normalizing the integral of a positive semidefinite function is always possible (if the endpoints of integration are specified), one can motivate multiple schemes for enforcing nonnegativity that result in different reconstructions $\hat{p}(z)$. We postpone to future work the exploration of adaptations of non-positive semidefinite representations and inclusion of the sparse basis representation in qp.

For each format, we address the following questions:

- When/where has the format appeared as a published catalog format, native photo- $z$ PDF code output format, and science application input format?

- What exactly is stored under the format, per galaxy (the parameters) and per catalog (the metaparameters)?

- What are the a priori strengths and weaknesses of the format?

\subsubsection{Regular Binning}

By far the most popular format for approximating and storing photo- $z$ PDFs is the piecewise constant step function, also called a histogram binning. It is the native output of a number of photo- $z$ PDF codes (Carrasco Kind \& Brunner 2014a; Sadeh et al. 2016; Cavuoti et al. 2017) and the only format that has been used for public release of photo- $z$ PDF catalogs (Sheldon et al. 2012; Tanaka et al. 2018; de Jong et al. 2017).

The metaparameters of the binned parametrization are the ordered list of redshifts $\vec{C}=\left(z_{1}, z_{2}, \ldots, z_{N_{f}}, z_{N_{f}+1}\right)$ serving as bin endpoints shared by all galaxies in the catalog, each adjacent pair of which is associated with a parameter $c_{i}=\int_{C_{i}}^{C_{i+1}} p(z) d z /\left(C_{i+1}-C_{i}\right)$. The qp histogram format assumes $p(z)=0$ when $z<C_{1}$ or $z \geq C_{N_{f}+1}$ and enforces the normalization condition ${ }^{13}$ $\sum_{i} c_{i}\left(C_{i+1}-C_{i}\right)=1$. The histogram format function $\mathcal{F}^{h}$ is thus the sum of a set of $N_{f}$ step functions, making

\footnotetext{
13 Note that this is not generally equivalent to the heuristic normalization condition $\sum_{i} c_{i}=1$ commonly enforced in public catalogs, such as the Sloan Digital Sky Survey's Data Release 8 (Sheldon et al. 2012). Unless redshift is treated as a discrete variable, the oversimplified normalization condition only holds if $C_{N_{f}+1}-C_{1}=N_{f}\left(C_{i+1}-C_{i}\right)$ for all $i$ (under a regular binning).
} 
the reconstructed estimator of the photo- $z \mathrm{PDF}$

$$
\hat{p}^{h}(z)=\sum_{i=1}^{N_{f}} c_{i} H\left(z-C_{i}\right) H\left(C_{i+1}-z\right)
$$

in terms of the Heaviside step function $H$ as the interpolating function $F$. Though qp supports arbitrary bin ends, here we only consider a regular binning, with $C_{i+1}=C_{i}+\delta$ for a constant $\delta=\left(C_{N_{f}+1}-C_{1}\right) / N_{f}$, as no irregular binning has yet been used for a public catalog of photo- $z$ PDFs.

In terms of performance as a photo- $z \mathrm{PDF}$ storage format, we should anticipate the regular histogram format to be wasteful in terms of information content; a photo- $z$ PDF with a very broad or compact PDF may have many parameters taking the same value $c_{i} \approx\left(C_{N_{f}+1}-C_{1}\right) / \delta$ or $c_{i} \approx 0$, for broad and compact PDFs respectively, that are redundant in storage. Additionally, we should expect the fidelity of $\hat{p}^{h}(z)$ to depend strongly on the bin widths relative to the sizes of and distances between features in the photo- $z$ PDFs.

\subsubsection{Random Samples}

Samples are often the native output format of machine learning algorithms due to the discrete nature of training sets (De Vicente et al. 2016). Such approaches by default typically produce large numbers of samples, far more than can realistically be stored by any survey, so are commonly compressed by subsampling (Hoyle et al. 2017). Samples are easy to use in standard science applications developed for redshift point estimates, so they have an established presence in the literature (Bonnett et al. 2016). The samples format of PDF storage appears elsewhere in astronomy, including the Gaia-ESO Survey's commitment to provide multi-dimensional PDFs of stellar parameters in the samples format (Bailer-Jones et al. 2013).

The parameters of the samples format are the $N_{f}$ samples $\vec{c}=\left(z_{1}, z_{2}, \ldots, z_{N_{f}-1}, z_{N_{f}}\right)$, where $C=N_{f}$ is an implicit metaparameter. The format function $\mathcal{F}^{s}$ that turns samples into a representation of the photo- $z$ PDF is simply the interpolator $F$. In the tests presented here, we use the Gaussian kernel density estimate (KDE) of scipy.stats.gaussian_kde. The samples representation is then

$$
\hat{p}^{s}(z)=\operatorname{KDE}_{C^{\prime}}(z ; \vec{c}) .
$$

Though samples are an obvious choice for photo- $z$ PDFs with narrow features of high amplitude, we expect that using a small number of samples from a broad photo- $z$ PDF may increase the variance of any ensemble metrics, as the sampling introduces additional shot noise. The researcher must also choose an interpolation method to reconstruct a photo- $z$ PDF from samples.

\subsubsection{Regular Quantiles}

One parametrization that has not previously been investigated in the context of photometric redshifts is that of quantiles, though they have appeared elsewhere in the astronomy literature (Sun et al. 2015; Pizzocaro et al. 2016; Laycock et al. 2017). The quantiles are defined in terms of the cumulative distribution function (CDF), which is the antiderivative of the PDF.

Under the quantile format, a photo- $z$ PDF catalog shares $N_{f}$ ordered CDFs $\vec{C}=\left(q_{1}, q_{2}, \ldots, q_{N_{f}-1}, q_{N_{f}}\right)$ where $0<q_{i}<1$ for all $i$. Each galaxy's catalog entry is the vector of redshifts $\vec{c}=\left(z_{1}, z_{2}, \ldots, z_{N_{f}-1}, z_{N_{f}}\right)$ satisfying $\operatorname{CDF}\left(c_{i}\right)=C_{i}$, so the quantile format function $\mathcal{F}^{q}$ is the derivative of an interpolation $F$ of the CDF. As with the samples representation, an interpolation function $F$ must be chosen for reconstructing the photo- $z$ PDF from the stored parameters. qp includes support for numerous scipy.interpolate options as well as straightforward linear interpolation.

Our interpolator $F$ in the tests presented here is the derivative of a spline function at $z_{1} \leq z \leq z_{N_{f}}$ and linear extrapolation subject to consistency with the definition of the CDF. The quantile representation implemented in this paper is thus

$$
\hat{p}^{q}(z)=\left\{\begin{array}{cc}
\frac{d}{d z}[F(z ; \vec{c})] & c_{1} \leq z \leq c_{N_{f}} \\
\hat{p}^{q}\left(c_{1}\right)\left(\frac{\hat{p}^{q}\left(c_{1}\right)}{2 C_{1}} z-1\right) & z<c_{1} \\
\hat{p}^{q}\left(c_{N_{f}}\right)\left(1-\frac{\hat{p}^{q}\left(c_{N_{f}}\right)}{2\left(1-C_{N_{f}}\right)} z\right) & z>c_{N_{f}+1}
\end{array}\right\} .
$$

In this study, we also restrict consideration to regular quantiles $C_{i} \equiv i /\left(N_{f}+1\right)$, though qp supports arbitrary quantile spacing.

The quantile parametrization (the namesake of the qp code) is expected to be an efficient approximation for photo- $z$ PDFs because it allocates storage evenly in the space of probability density. In contrast, the histogram format stores information evenly spaced in redshift, and the samples format stores information randomly in probability density. Depending on the native photo- $z$ PDF output format, converting to the quantile format may require $N_{f}$ numerical optimizations. We accelerate these optimizations by initializing at rough, approximate quantiles based on CDF evaluations on a grid.

\subsection{Comparison Metrics}

We aim to probe how closely photo- $z$ PDFs reconstructed from a limited set of stored parameters approx- 
imate the original, high-resolution representation $\hat{p}^{r}(z)$ of the reference catalog. This is done without knowledge of a galaxy's true redshift; there is, in fact, no notion of a true redshift in our analysis. (For a demonstration of how one might approach the distinct problem of evaluating the accuracy of a photo- $z$ PDF relative to a true redshift, see Polsterer et al. (2016), Schmidt et al. in preparation.)

We consider as a metric the loss of information incurred when using an approximation of the PDF $\hat{P}(z)$ instead of the reference PDF $P(z)$, given by the Kullback-Leibler divergence (KLD), which is defined as

$$
\operatorname{KLD}[\hat{P}(z) \mid P(z)]=\int_{-\infty}^{\infty} P(z) \log \left[\frac{P(z)}{\hat{P}(z)}\right] d z,
$$

where log is the natural logarithm throughout this paper unless otherwise indicated, such that the KLD is measured in nats (base $e$ digits, analogous to base 2 bits). Because there is in general no closed-form expression for the KLD, we calculate the discrete KLD

$$
\mathrm{KLD}[\hat{P}(z) \mid P(z)] \approx \delta_{f f} \sum_{z=z_{1}}^{z_{N_{f f}}} P(z) \log \left[\frac{P(z)}{\hat{P}(z)}\right]
$$

using evaluations of the PDF under each format on a very fine, regular grid $\left(z_{1}, z_{2}, \ldots, z_{N_{f f}-1}, z_{N_{f f}}\right)$ with resolution $\delta_{f f}=\left(z_{N_{f f}}-z_{1}\right) / N_{f f}$ for $N_{f f} \gg N_{f}$.

The most important feature of the KLD is its asymmetry: it is not a distance, like the root mean square error, that is the same from $P(z)$ to $\hat{P}(z)$ as it is from $\hat{P}(z)$ to $P(z)$. It is a divergence of the information lost when using $\hat{P}(z)$ to approximate $P(z)$. The KLD requires that both functions $P(z)$ and $\hat{P}(z)$ be PDFs (always positive semidefinite and integrating to unity); this may need to be explicitly enforced for some approximation formats. The KLD is always positive, and a smaller value indicates better agreement between the approximate representation $\hat{p}^{\mathcal{F}}(z)$ and the reference representation $p^{r}(z)$. In the Appendix, we review the properties of the KLD and establish some intuition for it.

Additionally, we consider the percent error

$$
\Delta_{m}[\hat{P} \mid P]=\frac{M_{m}[P]-M_{m}[\hat{P}]}{M_{m}[P]} \times 100 \%
$$

of the $m^{\text {th }}$ moment

$$
M_{m}[P]=\int_{-\infty}^{\infty} z^{m} P(z) d z \approx \delta_{f f} \sum_{z=z_{1}}^{z_{N_{f f}}} z^{m} P(z)
$$

of a PDF. We note that $M_{0}[P]=1$ for all properly normalized PDFs, $M_{1}[P]=\bar{z}$ is the mean, $M_{2}[P]$ is the variance, and $M_{3}[P]$ is the skewness. Though the first few moments are not in general sufficient to characterize a highly structured PDF, they are included in this analysis because they can prove useful in setting ballpark estimates of the influence of different systematics in various science cases.

The metrics considered in this paper are a subset of those included in the qp package and are specific to this investigation; different metrics may be more appropriate for other science use cases and indeed could lead to different format preferences.

\subsubsection{Individual photo-z PDF metrics}

Some science applications rely on the recovery of individual galaxy photo- $z$ PDFs that, for example, may be used as the basis for finding (Radovich et al. 2017) or constraining the masses of (Applegate et al. 2014) galaxy clusters. For this purpose, we calculate the KLD of each individual photo- $z$ PDF in our catalogs and then characterize the distribution of KLD values (which is itself a PDF) by its $m=1,2,3$ moments. We also calculate the percent error on the $m=1,2,3$ moments of each photo- $z$ PDF under all parametrizations for both ensembles. It is natural to expect the KLD distribution moments and moment percent errors to decrease with increasing $N_{f}$, indicating convergence toward Gaussian errors as the approximation improves. We use these aggregate statistics to quantify the fidelity of individual photo- $z$ PDF approximations for each dataset as a function of parametrization.

\subsubsection{Stacked $\hat{n}(z)$ estimator}

In addition to considering how the choice of storage parametrization affects the recovery of individual photo$z$ PDFs, we also demonstrate how one might use qp to choose the best parametrization for a particular science case. We encourage qp users to develop a metric around their own photo- $z$ PDF use cases, as the optimal parametrization may not be shared among all science applications of photo- $z$ PDFs.

In cosmology, photo- $z$ PDFs have thus far been used almost exclusively to estimate the redshift distribution function $n(z)$ necessary for calculating the correlation functions used by many cosmological probes (Clampitt et al. 2017; Hildebrandt et al. 2017). The most common way to estimate the redshift distribution function for a sample of $N_{g}$ galaxies is to average the photo- $z$ PDFs according to

$$
\hat{n}(z) \equiv \frac{1}{N_{g}} \sum_{k=1}^{N_{g}} \hat{p}_{k}(z)
$$


a procedure producing what we call the stacked estimator $\hat{n}(z)$ of the redshift distribution function (HarnoisDéraps et al. 2017; Hoyle et al. 2017). ${ }^{14}$ Note that to avoid introducing any preferred treatment between formats, Equation 9 uses the representation $\hat{p}(z)$ reconstructed from the stored parameters even when a given format may have a more efficient or principled way to obtain such an ensemble estimator directly from the stored parameters, e.g. adding the histogram components or combining all samples. While we do not recommend the approach of Equation 9 to estimate the redshift distribution (see Choi et al. (2016) for justification and Malz et al., in preparation for alternative methods), we use it here to generically demonstrate how one would optimize the choice of photo- $z$ PDF parametrization in the context of a familiar science application.

As the stacked estimator is normalized so that it, too, is a PDF, the KLD from the approximation (the stacked estimator of a catalog of evaluations of reconstructed photo- $z$ PDFs) to the original (the stacked estimator of a catalog of evaluations of the high-resolution reference photo- $z$ PDFs) serves as a metric for a specific science use case of photo- $z$ PDFs. Because the accuracy of lower-order moments of the redshift distribution function dominates the weak lensing error budget, we also compare the percent error on the $m=1,2,3$ moments of $\hat{n}(z)$. However, this information may be less relevant due to the broad range of redshifts and small number of galaxies considered in each instantiation. Furthermore, we note that the dominance of the first few moments of $\hat{n}(z)$ may not always hold true as the methodology of photo- $z$ PDF usage in cosmology evolves.

\section{PHOTO-Z TEST DATA}

With the expectation that the optimal parametrization for approximating photo- $z$ PDFs may differ according to the properties of the original photometric data, we demonstrate a procedure for vetting photo- $z$ PDF parametrizations on a pair of mock datasets, each intended to be realistic predictions of subsets of the anticipated LSST photo- $z$ PDFs. All photo- $z$ PDFs are fit to simulated LSST 10-year ugrizy apparent magnitudes and errors (Ivezić et al. 2008) using the publicly available Bayesian Photometric Redshift (BPZ) code (Benítez 2000), which employs fitting to a library of spectral energy distribution (SED) templates. The choice of photo$z$ PDF estimation method, however, is not relevant to

\footnotetext{
${ }^{14}$ Equation 9 is sometimes modified by weights specific to each galaxy based on the relative prevalence of galaxies with similar photometry in a reference population (Sheldon et al. 2012; Tröster et al. 2017)
}

this study; so long as the mock photo- $z$ PDFs are realistically complex, meaning they take shapes with features comparable to those we expect to see in photo- $z$ PDFs from real datasets with similar photometric properties, it does not matter whether the photo- $z$ PDFs produced by $\mathrm{BPZ}$ are accurate redshift posteriors. We seek only to optimize the fidelity of the stored photo- $z$ PDF relative to the original photo- $z$ PDF from a representative photo- $z$ PDF fitting code. (See Tanaka et al. 2018; de Jong et al. 2017; Amaro et al. 2016, Schmidt et al. in preparation for other work comparing the accuracy of photo- $z$ PDFs produced by different methods.) As BPZ is a widely used and well established method, we assume that the photo- $z$ PDFs produced by it are of representative complexity. The default format of BPZ is a $N_{f f}>200$ gridded parametrization with resolution exceeding the available storage for an LSST-like survey. Because we believe that each galaxy has an underlying redshift interim posterior probability density that is a continuous function, to which the output of BPZ is itself a high-resolution approximation in the form of evaluations on a grid, we fit each gridded photo- $z$ PDF with a Gaussian mixture model that we designate as the reference representation $p^{r}(z)$ for our tests. The number of components of the mixture model is rounded up from the $99^{\text {th }}$ percentile of the modality distribution of the photo- $z$ PDF catalog in question.

\subsection{Bright data mock catalog}

Our first dataset is an $N_{g}=10^{5}$ object subset of the Graham et al. (2018) simulated galaxy catalog used for LSST photometric redshift experiments. The data builds on the Millennium simulation of large-scale structure (Springel et al. 2005), the galaxy formation models of Gonzalez-Perez et al. (2014), and the lightcone construction techniques of Merson et al. (2013). We use the software of Connolly et al. (2014) to derive observed apparent ugrizy magnitudes from the true apparent magnitudes and 10-year LSST observational errors of Ivezić et al. (2008) before imposing cuts in true redshifts $z<3.5$ and observed apparent magnitudes fainter than the predicted 10-year limiting magnitudes in each filter $(u<26.1, g<27.4, r<27.5, i<25, z<26.1$, and $y<24.9)$ to realistically simulate non-detections.

The photo- $z$ PDF estimates for this simulated catalog use BPZ templates based on the VIsible MultiObject Spectrograph Very Large Telescope Deep Survey set of spectra (Fèvre et al. 2005), as in Ilbert et al. (2006). This catalog also uses the default parameter settings for BPZ with the two additions of a photometric redshift maximum of 3.5 and an $i$-band magnitude prior. The photo- $z$ PDFs from BPZ are in the form of $N_{f f}=351$ 

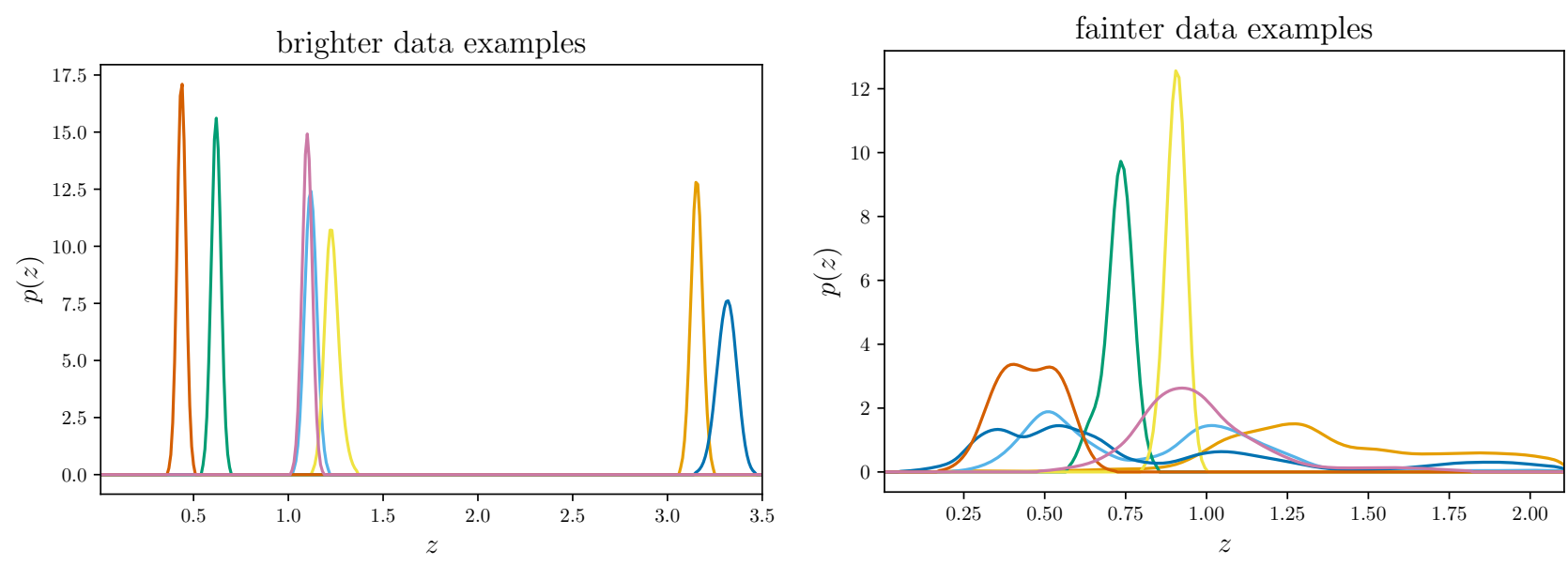

Figure 2. Example photo- $z$ PDFs from the two mock LSST datasets. Left: The bright mock photometry yields largely narrow, unimodal photo- $z$ PDFs. Right: The faint mock photometry contains a higher proportion of broad and/or multimodal photo- $z$ PDFs.

evaluations of the probability density on a regular grid of redshifts $0.01<z<3.51$, a subsample of which are shown in the left panel of Figure 2. As the figure shows, the photo- $z$ PDFs from this dataset tend to be unimodal and sharply peaked, as if coming from brighter photometric data due to the conservative cuts in photometric magnitudes of this dataset. The brighter catalog reference photo- $z$ PDFs are three-component Gaussian mixtures fit to this data.

\subsection{Faint data mock catalog}

Our second dataset is an independent simulation of the expected LSST galaxy sample, the Buzzard-highresv1.0 mock galaxy catalog of deRose et al. in preparation of galaxies with SEDs drawn from an empirical library of $\sim 5 \times 10^{5}$ SEDs from the Sloan Digital Sky Survey (SDSS). Given an SED, redshift, and absolute $r$-band magnitude for each galaxy, the ugrizy magnitudes are derived from the aforementioned 10-year LSST errors. The catalog contains $N_{g} \approx 10^{5}$ galaxies $z<2.105$ to a depth of $i<26.9,1.5$ magnitudes deeper than the expected LSST gold sample of galaxies (LSST Science Collaboration 2009), that will have $S / N \gtrsim 30$ in multiple bands.

We use a custom BPZ prior using a subset of the Buzzard-highres-v1.0 catalog and a spanning template set via a simple $\mathrm{k}$-means clustering algorithm based on 100 of the SDSS SEDs used to create the Buzzard catalog. BPZ produces photo- $z$ PDFs in the format of probability density evaluations on a regular grid of $N_{f f}=211$ redshifts $0.005 \leq z \leq 2.105$, a subsample of which are plotted in the right panel of Figure 2. The exceptional depth and known degeneracies (e. g. the Lyman/Balmer break degeneracy) lead us to expect the presence of multimodal photo- $z$ PDFs observed in the figure. The fainter catalog reference photo- $z$ PDFs are five-component Gaussian mixtures fit to this data.

\section{RESULTS \& DISCUSSION}

We evaluate the metrics of Section 2.2 on 10 random instantiations of catalogs of $N_{g}=100$ galaxies drawn randomly from each of the datasets discussed in Section 3 and with each of $N_{f}=3,10,30,100$ stored parameters for the three formats of Section 2.1. Our analysis characterizes the results using the median and interquartile range (IQR), with lower and upper bounds representing the $25^{\text {th }}$ and $75^{\text {th }}$ percentiles, as a modelindependent error characterization appropriate for our small number of mock catalogs. We then illustrate how our results could be used to choose an appropriate parametrization for each dataset given constraints on the distribution of KLDs or moment percent errors of individual photo- $z$ PDFs, the KLD or moment percent error of a science metric $(\hat{n}(z)$ in this case), or the available storage capacity.

\subsection{Individual photo-z PDFs}

We compare our three formats on the basis of the distributions of the KLD calculated for every photo- $z$ PDF in the two datasets. An example of an individual photo$z$ PDF KLD distribution for the bright dataset with $N_{f}=10$ is shown in Figure 3.

To distill what is observed in the ten instantiations of plots like Figure 3 for both datasets and all parametrizations, we compare the first three moments of the distributions of metric values for the distribution of the KLDs of individual photo- $z$ PDFs under each parametrization, summarized in Figure 4. While it is obvious that one 


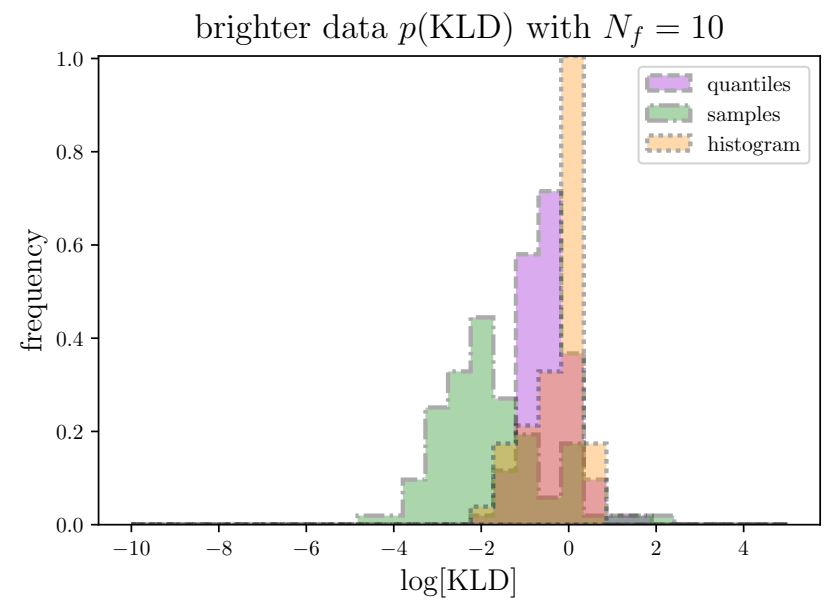

Figure 3. The distribution of log-KLD values for $N_{g}=100$ photo- $z$ PDFs from the bright dataset with $N_{f}=10$ over the quantiles (purple with dashed border), samples (green with dash-dotted border), and histogram (orange with dotted border) formats. In this instantiation, the samples format has a lower median KLD than the quantiles format, which has a lower median KLD than the piecewise constant format. Note that the distributions are over log-KLD, so the ordering of the formats by the breadth of the log-KLD distribution is the same as the order by the median.

would like the mean of the KLD distribution to be low, interpretation of higher-order moments is less clear. In a science application that is robust to photo- $z$ PDF outliers, a parametrization with a high variance or skewness may be acceptable, whereas another science application that simply requires a well-characterized error distribution may tolerate a higher mean in exchange for lower variance and skewness. To meaningfully interpret the KLDs of individual photo- $z$ PDFs, it will be necessary for those using photo- $z$ PDFs in their science to calculate the requirements on the acceptable degree of information loss.

As expected, the behavior of the log-moments of the $\log$-KLD distribution is highly correlated for a given format and number of parameters, for both datasets. The bright dataset has slightly higher log-KLD log-moments than the faint dataset at all $N_{f}$ and across all formats, meaning information loss is enhanced for more strongly featured data; this observation is not surprising because the narrow, unimodal photo- $z$ PDFs of the bright dataset have long tails of very low probability that are emphasized by the KLD. Both datasets exhibit decreasing moments for the quantile and samples formats as $N_{f}$ increases, though the marginal improvement at high $N_{f}$ is greater for the faint dataset.

The log-KLD log-moments are higher for quantiles than for samples in both datasets, except at $N_{f}=100$ for the bright dataset. The histogram format's log-KLD $\log$-moments are higher than those of other formats at the lowest $N_{f}$ and steadily decrease in a manner similar to the other formats, except at the highest $N_{f}$ values where the histogram format's log-KLD log-moments decrease much more quickly. Neither of these results are unexpected because of the KLD's sensitivity to the tails; our choise of PDF reconstruction method for the quantile format is most susceptible to error in the tails of the PDF, and only the histogram format preserves information uniformly at all redshifts rather than at all probabilities.

In Figure 5, we also examine the percent error on the first three moments of the photo- $z$ PDFs under each approximation, using the base-10 log for interpretability.

Note that the error bars here are larger because they include the effects of variation between individual galaxies, whereas all other plots of moments have error bars that represent only the variation due to catalog instantiations.

Though the $\log _{10}$-percent error of the moments of individual photo- $z$ PDFs also exhibits significant correlation between the moments for a given parametrization, the behavior is otherwise markedly different from that of the log-moments of the photo- $z$ PDF ensemble's logKLD distribution. The percent errors of the moments of the approximate photo- $z$ PDFs are overall lower in the bright dataset than those of the faint dataset over the same range of number of stored parameters; this is expected because there is simply less information to capture in the bright dataset. The unwaveringly linear marginal improvement in the log-percent error of the per-galaxy photo- $z$ PDF moments with increasing log$N_{f}$ may reflect the fact that samples are taken randomly in the space of probability and thus unaffected by the interpolation artifacts of the quantile format and the interplay between the scales of features and bins of the histogram format.

For the bright dataset, the quantile format is the only one that consistently achieves sub-percent errors in photo- $z$ PDF moments even at low $N_{f}$. Furthermore, for the bright dataset, the quantile format minimizes the percent error at all $N_{f}$, whereas the samples format outperforms the histogram format at low $N_{f}$ before the histogram format overtakes it at high $N_{f}$. Again, this behavior is expected of the narrow, unimodal photo$z$ PDFs of the bright dataset because large histogram bins are ineffective at capturing small-scale structure and including more samples does not significantly improve preservation of such features.

The qualitative behavior of the moment percent error of all formats is the same for the faint dataset as that 

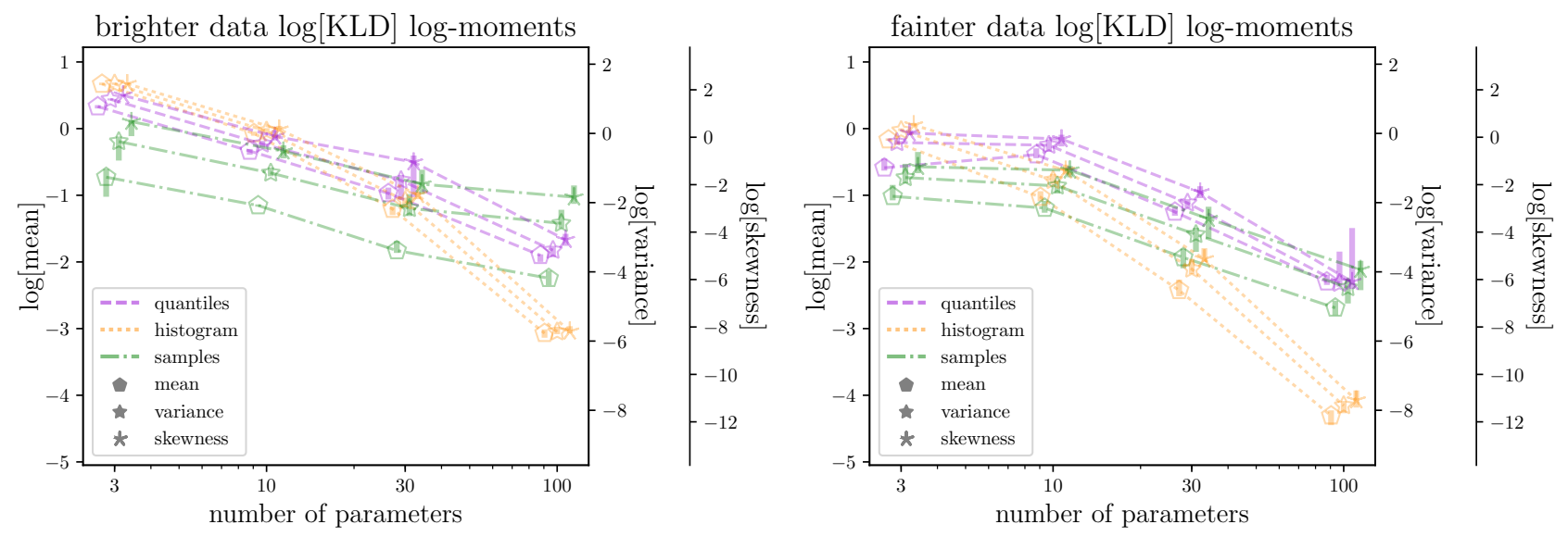

Figure 4. The medians of the mean $(\bullet)$, variance $(\star)$, and skewness $(\star)$ of the log-KLD distributions for each dataset as a function of the number $N_{f}$ of stored parameters for the quantile (purple dashed line), samples (green dash-dotted line), and histogram (orange dotted line) formats with IQR error bars based on 10 instantiations of 100 galaxies, which are offset about $N_{f}$ to improve readability. Left panel: The moments of the distribution of individual photo- $z$ PDF log-KLD values of the bright mock catalog are minimized when they are stored as samples, except at large $N_{f}$. Right panel: The faint mock catalog achieves equivalence of the formats in the moments of the log-KLD distributions at a much lower $N_{f}$, ultimately showing the histogram format minimizes the moments at all but the smallest $N_{f}$.

brighter data $\hat{p}(z)$ moment $\log _{10}$-percent errors

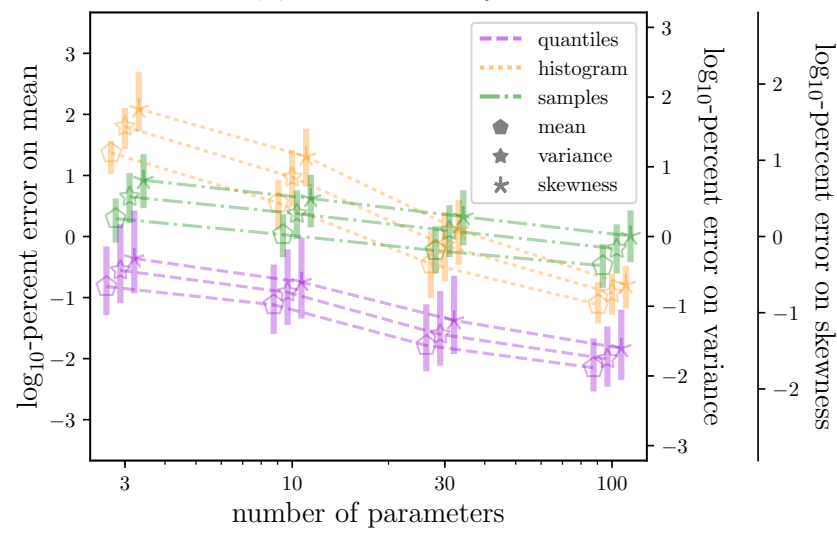

fainter data $\hat{p}(z)$ moment $\log _{10}$-percent errors

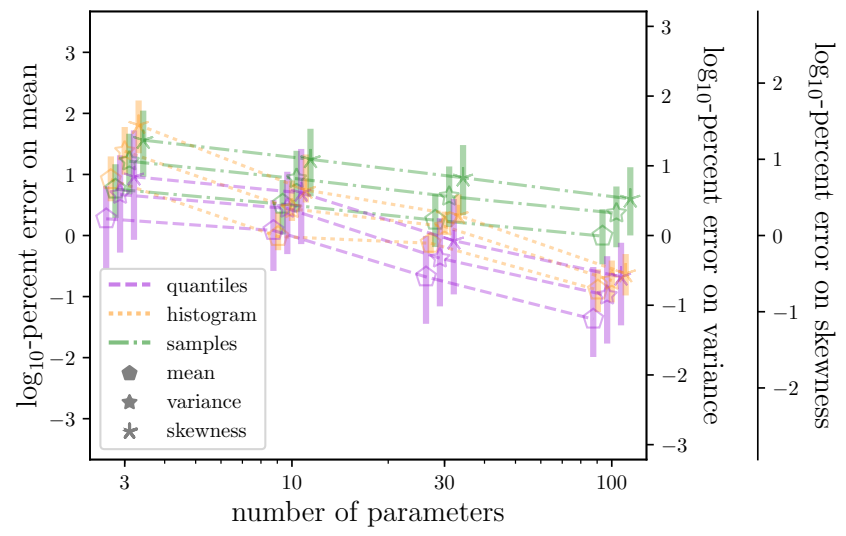

Figure 5. The median $\log _{10}$-percent errors on the mean $(\triangle)$, variance $(\star)$, and skewness $(\star)$ of the photo- $z$ PDFs for each dataset as a function of the number $N_{f}$ of stored parameters per photo- $z$ PDF for the quantile (purple dashed line), samples (green dash-dotted line), and histogram (orange dotted line) formats with IQR error bars based on 10 instantiations of 100 galaxies, where the $\log _{10}$-percent errors and their IQRs are offset about $N_{f}$ to improve readability. Left panel: The bright photo- $z$ PDF ensemble's moment percent errors are minimized by the quantile format at all $N_{f}$. Right panel: The faint photo- $z$ PDF ensemble's moment percent errors are high for all formats at low $N_{f}$ but distinct at high $N_{f}$, with the quantile format overall outperforming the samples and histogram formats.

of the bright dataset at $N_{f}=3$. In the faint dataset, the inclusion of $N_{f}=30$ parameters decreases the moment percent error of the histogram format more significantly than the quantile or samples formats, to the point that the histogram and quantile formats have comparable moment percent errors. At higher $N_{f}$ in the faint dataset, the quantile and histogram formats continue to improve faster than the samples format, with the percent errors on the photo- $z$ PDF moments being consis- tently lower for the quantile format than for the histogram format. The broad, multimodal photo- $z$ PDFs of the faint dataset enable achievement of sub-percent accuracy in the moments only with $N_{f} \geq 30$ under the quantile format and $N_{f}=100$ with the histogram format.

\subsection{Stacked $\hat{n}(z)$ estimator}




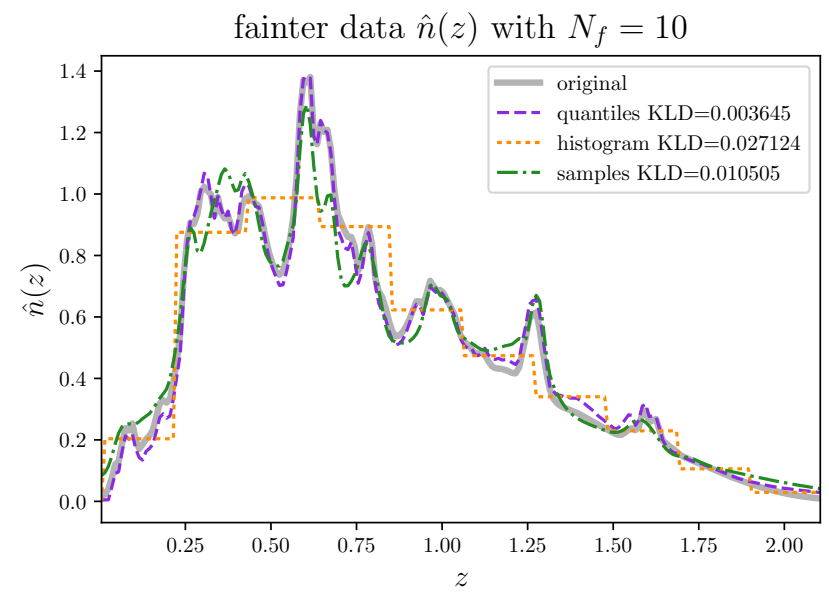

Figure 6. An example of the stacked estimator of the redshift distribution, for a subsample of $N_{g}=100$ galaxies drawn from the faint data mock catalog and with $N_{f}=10$ parameters used for each photo- $z$ PDF; the small-scale features are due to the small number of galaxies in the sample. The most striking characteristic of $\hat{n}(z)$ of a small number of galaxies with a relatively small number of parameters is the coarseness of the histogram format (orange dotted line) relative to the quantile (purple dashed line) and samples (green dash-dotted line) formats, both of which are fairly close to $\hat{n}(z)$ derived from evaluating the original, high-resolution photo- $z$ PDFs (thick gray line).

Figure 6 shows an example of $\hat{n}(z)$ of photo- $z$ PDFs reconstructed from just $N_{f}=10$ parameters under each of our three approximation formats, evaluated on the same fine grid as the input photo- $z$ PDFs. The strong features in the curve are due to the small sample size of $N_{g}=100$ galaxies. As expected, the stacked histogram is quite coarse because of the step function interpolation, while the stacked estimator of the redshift distribution based on photo- $z$ PDF representations that are interpolations of stored samples and quantiles are much closer to the stacked estimator of the original, high-resolution photo- $z$ PDFs. The KLD for each format is also included in the plot; in this instance, the KLD is lowest for the quantile format and highest for the histogram format.

Again, due to the variation between $N_{g}=100$ galaxy subsamples, we repeat 10 times the procedure that produced Figure 6 to generate a distribution over the KLD of the stacked estimator of the redshift distribution for each format and dataset. The $\hat{n}(z) \mathrm{KLD}$ values for each parametrization on both mock datasets are collected and plotted in Figure 7, with error regions based on the IQR of the 10 instantiations.

Figure 7 shows that the two datasets clearly share some features:
1. As expected, the KLD drops as the number of stored parameters increases, for all formats.

2. The quantile format minimizes the KLD at all numbers of stored parameters considered.

3. The histogram format leads to substantial loss of information relative to the other formats except at large numbers of stored parameters where it is on par with the samples format.

However, there are also ways in which the behavior of the KLD on $\hat{n}(z)$ differs due to the data quality's significant impact on this metric:

1. The faint dataset in general achieves lower KLD values than the bright dataset, likely a consequence of the strong features present in $\hat{n}(z)$ for the bright dataset in our subsamples of 100 galaxies.

2. The rate of marginal improvement in the KLD of $\hat{n}(z)$ with increasing $N_{f}$ is lower in the bright dataset than the faint dataset; in other words, saving more parameters may have a greater marginal benefit for the faint dataset than for the bright dataset.

3 . The $\hat{n}(z)$ KLD of the samples format is not substantially higher than that of the quantile format in the faint dataset but is for the bright dataset, which may reflect the subjectivity of the reconstruction scheme used for those two formats.

We also address the relative, marginal, and absolute performance and consistency thereof of the KLD on $\hat{n}(z)$ for each parametrization as a function of format and $N_{f}$ for each dataset. To guide this process, we interpret Figure 7 in the context of constraints on storage allocation imposed by the survey and constraints on the acceptable degree of information loss imposed by the science requirements, which we anticipate establishing in the future.

A constraint on storage resources corresponds to a vertical line at a given $N_{f, \lim }$ in Fig. 7 ; the best format would be the one that achieves the lowest KLD at $N_{f, \text { lim }}$. For example, if $N_{f, \lim }=10$ stored parameters, the quantile format would be optimal for the bright dataset because it has the lowest KLD value by a large margin compared to other formats. If the faint dataset were subject to the same constraint, the quantile and samples formats could both be good candidates for a storage parametrization, with the quantile format opening the possibility of a lower KLD. 

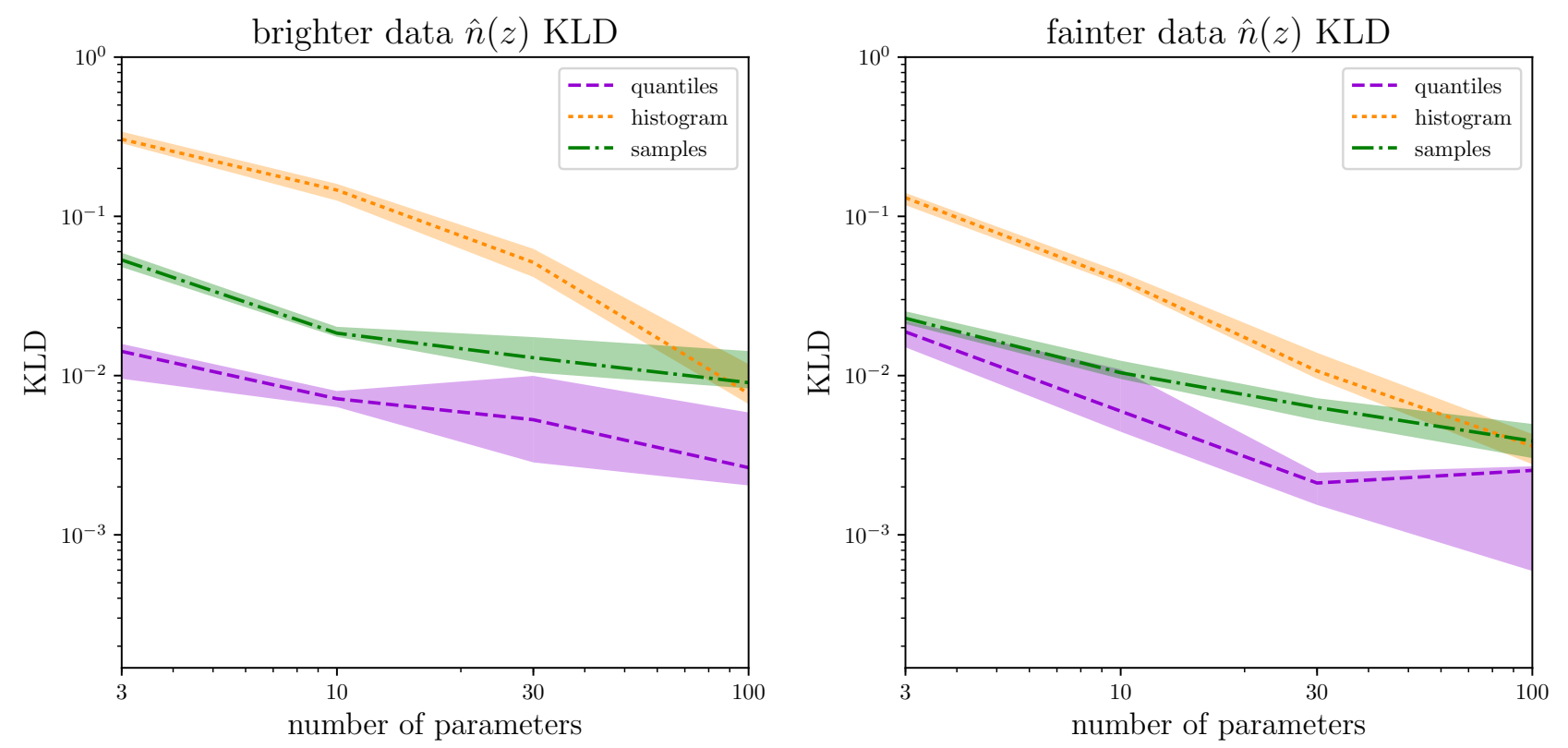

Figure 7. The KLD between $\hat{n}(z)$ derived from approximate photo- $z$ PDF representations and $\hat{n}(z)$ derived from the original, high-resolution photo- $z$ PDFs, as a function of number $N_{f}$ of stored parameters, for the quantiles (purple dashed line), samples (green dash-dotted line), and histogram (orange dotted line) formats. Shaded regions indicate the IQR errors derived from 10 subsamples of 100 galaxies and lines indicate the median of the distribution. Left panel: The bright photo- $z$ PDF catalog's KLD of $\hat{n}(z)$ is minimized by the quantile format at all $N_{f}$. Right panel: The faint photo- $z$ PDF catalog's KLD of $\hat{n}(z)$ is minimized by the quantile format at all $N_{f}$, though the samples format's performance is comparable.

A constraint on the acceptable loss of information due to compression and reconstruction of photo- $z$ PDFs corresponds to a horizontal line at some $\mathrm{KLD}_{\text {lim }}$ in Figure 7; the best parametrization would correspond to the format that achieves $\mathrm{KLD}_{\text {lim }}$ at the lowest $N_{f}$. For example, if our science requires $\mathrm{KLD}_{\lim }=10^{-2}$ nats, the optimal parametrization would be quantiles with $N_{f}=3$ for the bright dataset and quantiles with $N_{f}=10$ for the faint dataset.

If there is some flexibility in the allocation of storage for photo- $z$ PDFs, as is the case for LSST, it is valuable to examine the asymptotic behavior of the KLD as a function of the number of stored parameters for each format considered. It may be possible to request additional storage resources for the survey's photo- $z$ PDFs if the KLD is significantly reduced with a slightly larger $N_{f}$.

We also calculate the percent error on the moments of the stacked estimator of the redshift distribution, as these may be more useful for understanding error propagation in cosmology due to photo- $z$ PDF storage parametrization than the KLD, for which no such infrastructure yet exists. The percent error on the first three moments of the stacked estimator of the redshift distribution function is shown in Figure 8, and it is clear that the photometric data quality dominates this metric.
To explain this, we draw attention to Figure 6 and note that, though the true distribution of redshifts is similar, the redshift range over which they are defined is larger for the bright dataset than the faint dataset.

In the bright dataset, the evolution of the $\hat{n}(z)$ moment errors with $N_{f}$ differs for the histogram format relative to the samples and quantile formats, which exhibit essentially no evolution in excess of the error bars between instantiations. For $N_{f}=3$, the histogram format's moments are grossly underestimated because most of the probability density of $\hat{n}^{r}(z)$ falls into the lowest redshift bin, and most of the probability density of $\hat{n}^{r}(z)$ is above the middle of that bin. When the bins are too small, at $N_{f}=100$, those at high redshifts have most of their probability density below the middle of the bin, leading to slightly overestimated moments. Because the photo- $z$ PDFs in the bright dataset are so narrow and unimodal overall, the reconstructions of the samples and quantile parametrizations are highly accurate where most of the probability density is, even with low $N_{f}$, so the reference representation moments are consistently recovered to within $<1 \%$.

In the faint dataset, the issues are different because the redshift range of the original photo- $z$ PDFs is smaller and the photo- $z$ PDFs themselves are broader. The samples format has no significant evolution in moment 
brighter data $\hat{n}(z)$ moment percent errors

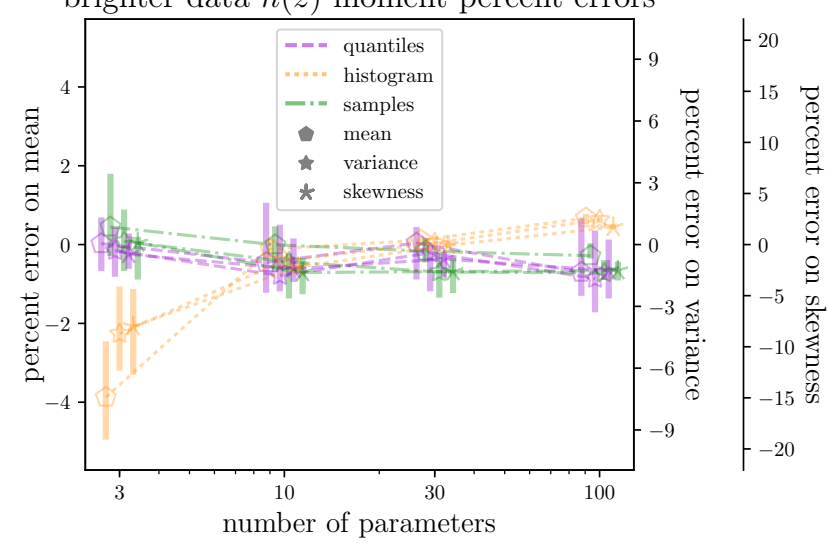

fainter data $\hat{n}(z)$ moment percent errors

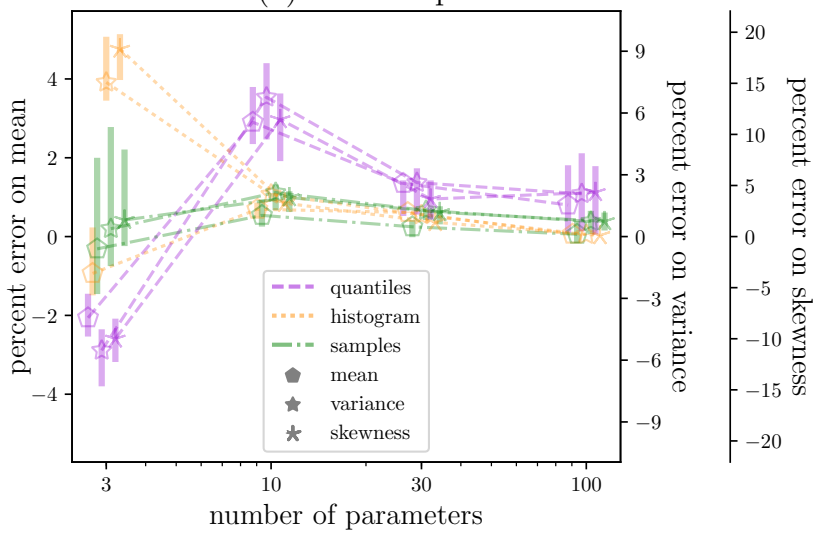

Figure 8. The median percent error on the mean $(\triangle)$, variance $(\star)$, and skewness $(\star)$ of the stacked estimator $\hat{n}(z)$ of the redshift distribution for each dataset as a function of the number $N_{f}$ of stored parameters for the quantile (purple dashed line), samples (green dash-dotted line), and histogram (orange dotted line) formats with IQR error bars based on 10 instantiations of 100 galaxies, where the percent errors and their error bars are offset about $N_{f}$ to improve readability. Left panel: The bright dataset shows evolution with $N_{f}$ of the $\hat{n}(z)$ moment percent errors for the histogram format but none for the samples and quantile formats. Right panel: The faint dataset shows qualitatively different evolution with $N_{f}$ of the $\hat{n}(z)$ moment percent errors for the three formats and for each moment.

errors with $N_{f}$, the histogram format severely overestimates the higher moments at low $N_{f}$, and the quantiles format severely underestimates the moments at low $N_{f}$, severely overestimates them at intermediate $N_{f}$, and moderately overestimates them at high $N_{f}$. The samples format may suffer from shot noise for broad, multimodal photo- $z$ PDFs, but the result is just spikier photo- $z$ PDFs that produce narrow features in $\hat{n}(z)$ that do not significantly affect the moments, explaining the minimal impact on the percent errors of the moments of $\hat{n}(z)$. The histogram format's overestimation of the variance and skewness at low $N_{f}$ in the faint dataset is caused by the bulk of the probability density of $\hat{n}^{r}(z)$ falling almost evenly into the two low redshift bins with far less probability in the highest bin.

As was hinted at in Figure 4, the quantile parametrization's photo- $z$ PDF KLD distribution has large moments, and the KLD is most sensitive to a poor approximation of the tails of the distribution. Both the underestimation of the $\hat{n}(z)$ moments at low $N_{f}$ and the overestimation of the $\hat{n}(z)$ moments at intermediate $N_{f}$ are due to the choice of a suboptimal reconstruction scheme for quantiles that could doubtlessly be improved in the future. The quantile format's overestimation of the moments even at high $N_{f}$ can be explained by the fact that qp does not limit the quantile values to the redshift range over which the original photo- $z$ PDFs were defined. A broad photo- $z$ PDF may thus be reconstructed with probability density outside the redshift range of the original photo- $z$ PDFs and then truncated and normalized prior to calculating the KLD.
Because broad photo- $z$ PDFs are more likely to occur at high redshift, this excess probability is more likely to be at high redshift, slightly but consistently inflating all moments.

\section{CONCLUSIONS \& FUTURE DIRECTIONS}

This work develops a principled, systematic approach to choosing a parametrization for storing a catalog of photo- $z$ PDFs from a survey of known data properties with a goal of balancing the available storage resources against the accuracy of the photo- $z$ PDFs and science products thereof reconstructed from the stored parameters. We demonstrate the recommended method on two realistic mock datasets representative of upcoming photo- $z$ PDF catalogs and draw the following conclusions:

- Some general trends are shared among the datasets we used in our tests, but much of the qualitative and quantitative behavior is different. The properties of the photo- $z$ PDF catalog influence the optimal compression scheme.

- The parametrization that best approximates individual photo- $z$ PDFs may differ from the parametrization that optimizes a given science metric. The science goals must motivate the metric that guides the choice of parametrization.

- In our LSST-like examples with metrics motivated by gravitational lensing probes of cosmology, we confirm the expectation that regular binning and 
uniform sampling in the space of probability is more effective than regular binning in redshift. This trend can only be enhanced as the quantile and sample reconstruction schemes improve.

To be clear, we do not advocate for a one-size-fitsall solution to the problem of compressing photo- $z$ PDF catalogs and emphasize that any decision should be motivated by science requirements and account for the absolute, relative, and marginal behavior of the formats considered as a function of the number of stored parameters.

For the case of LSST, though the histogram format has the strongest presence in the photo- $z$ PDF literature, it exhibits a higher loss of information and moment percent error of the reconstructed photo- $z$ PDFs, except when a very large number of parameters are stored, so we do not recommend its use for LSST's photo- $z$ PDF catalog. Given the constraint that LSST will be able to store only $\sim 100$ numbers to describe the redshift of each galaxy and intends to include the output of several photo- $z$ PDF codes, we can safely say that LSST can store the output of more than one photo- $z$ PDF code without risk of significant loss of information. Had our results indicated a significant improvement in our metrics for a small increase in the number of stored parameters, we would present to decision-makers within the collaboration evidence in support of increasing that allocation.

Furthermore, though we discussed the previous use of each format in science calculations, we do not endorse any format on the basis of existing infrastructure for a particular science application. Rather, we anticipate great advances in the development of analysis techniques that best make use of the information in photo$z$ PDFs and encourage the community to then choose parametrizations that most effectively serve the needs of those intended practices. Future analyses may also consider options we did not, such as additional formats, new metrics, variable $N_{f}$ over the PDF ensemble, irregular spacing of shared parameters $\vec{C}$, and improved samples and quantile reconstruction procedures.

So that decisions of this kind can be optimized for all future surveys, the qp Python package developed for this project is made public on GitHub as a tool for use by the broader community. We invite contributions of formats, metrics, and reconstruction schemes to the public GitHub repository.

\section{APPENDIX}

We develop some intuition for the Kullback-Leibler Divergence by contrasting it with the familiar metric of

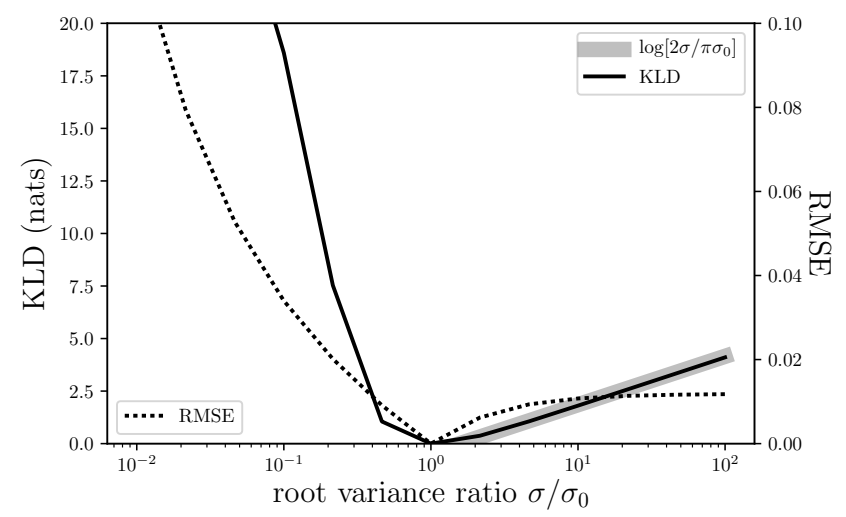

Figure 9. The KLD and RMSE as a function of the root variance ratio $r$ for a simple Gaussian example. The KLD (solid line) rises sharply at $\sigma<\sigma_{0}$ and is proportional to the $\log$ of the inverse precision $r$ for $\sigma>\sigma_{0}$, behavior that is qualitatively similar to that of the RMSE (dotted line).

the root-mean-square error (RMSE)

$$
\mathrm{RMSE}=\sqrt{\int(P(z)-\hat{P}(z))^{2} d z} .
$$

Consider the simple example of a Gaussian $P(z)=$ $\mathcal{N}\left(\mu_{0}, \sigma_{0}^{2}\right)$ being approximated by a Gaussian $\hat{P}(z)=$ $\mathcal{N}\left(\mu, \sigma^{2}\right)$, whose KLD is

$$
\mathrm{KLD}=\frac{1}{2}\left(\log \left[\frac{\sigma^{2}}{\sigma_{0}^{2}}\right]+\frac{\sigma_{0}^{2}}{\sigma^{2}}+\frac{\left(\mu-\mu_{0}\right)^{2}}{\sigma^{2}}-1\right)
$$

To get a sense of the units of information, we can calculate the KLD and RMSE in some limiting cases. If $\sigma=\sigma_{0}$ but $\mu=\mu_{0}+1$, we obtain KLD $=\frac{1}{2}$ nat - if the mean of the approximation is wrong by an additive factor of $\sigma$, half a nat of information is lost. If $\mu=\mu_{0}$ but $\sigma=\sqrt{2 \pi} \sigma_{0}$, we find $\mathrm{KLD} \approx \frac{1}{2}$ nat - half a nat of information is also lost if the variance of the approximation is off by a multiplicative factor of $2 \pi$.

We can use the KLD to identify notions of imprecision and inaccuracy. Intuitively, precision must be related to how close $\sigma$ is to $\sigma_{0}$ and accuracy must be related to how close $\mu$ is to $\mu_{0}$.

If $\mu \approx \mu_{0}$, we can say $\mathrm{KLD} \sim \log [r]+\frac{1}{2} r^{-2}-\frac{1}{2}$ where $r^{-1} \equiv \frac{\sigma_{0}}{\sigma}$ is a measure of precision, whose behavior is illustrated in Figure 9, alongside that of the RMSE. We observe that an overestimated variance increases the KLD as the log of the square root of the ratio of the estimated variance to the true variance.

When $\sigma \approx \sigma_{0}$, KLD $\sim t^{2}$ in terms of the tension $t \equiv$ $\frac{\left(\mu-\mu_{0}\right)^{2}}{\sigma^{2}}$, whose concordance is illustrated in Figure 10. There is some limiting tension $t_{\mathrm{lim}} \approx 2$ below which the RMSE is more sensitive than the KLD and above which the KLD is more sensitive than the RMSE. This 


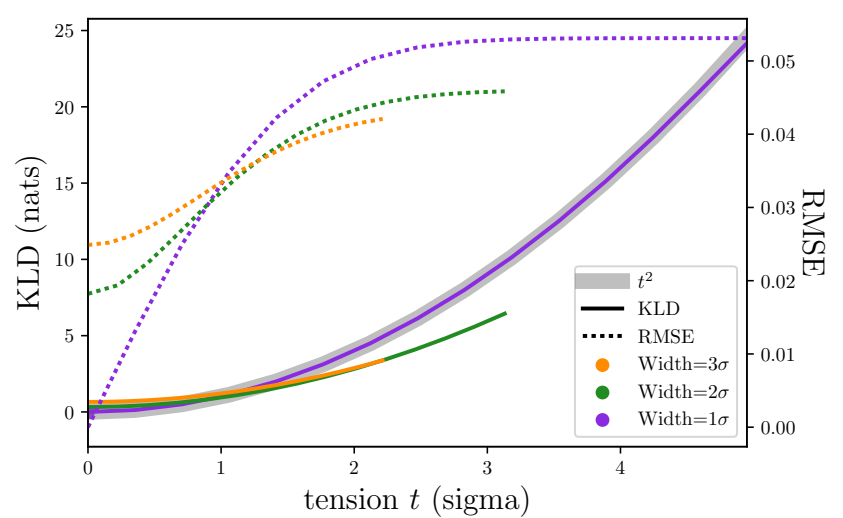

Figure 10. The KLD and RMSE as a function of the tension $t$ for a simple Gaussian example. The KLD (solid lines) is equal to the square of the tension $t$, with a small offset when $r \neq 1$, whereas the RMSE (dotted lines) is relatively insensitive to tension past a certain point but more sensitive to $r \neq 1$.

behavior hints at the KLD's reputation for sensitivity to the tails of the reference PDF. The notion of tension may be more important for cosmological applications of photo- $z$ PDFs, indicating the KLD may be a more appropriate metric for coarser approximations and the RMSE may be a more appropriate metric for less coarse approximations.

\section{ACKNOWLEDGMENTS}

This work was incubated at the 2016 LSST-DESC Hack Week hosted by Carnegie Mellon University. AIM is advised by David W. Hogg and was supported by National Science Foundation grant AST-1517237. The work of AIM was also supported by the U.S. Department of Energy, Office of Science, Office of Workforce Development for Teachers and Scientists, Office of Science Graduate Student Research (SCGSR) program, administered by the Oak Ridge Institute for Science and Education for the DOE under contract number DESC0014664. The work of PJM was supported by the U.S. Department of Energy under contract number DEAC02-76SF00515. SJS was partially supported by the National Science Foundation under grant N56981CC.

We would like to thank Chad Schafer, Chris Morrison, Boris Leistedt, Stefano Cavuoti, Maciej Bilicki, Johann
Cohen-Tanugi, Eric Gawiser, Daniel Gruen, and Seth Digel for helpful feedback in the preparation of this paper. AIM thanks Coryn Bailer-Jones, Eric Ford, and Morgan Fouesneau for providing context for this work outside cosmology.

The DESC acknowledges ongoing support from the Institut National de Physique Nucléaire et de Physique des Particules in France; the Science \& Technology Facilities Council in the United Kingdom; and the Department of Energy, the National Science Foundation, and the LSST Corporation in the United States. DESC uses resources of the IN2P3 Computing Center (CC-IN2P3Lyon/Villeurbanne - France) funded by the Centre National de la Recherche Scientifique; the National Energy Research Scientific Computing Center, a DOE Office of Science User Facility supported by the Office of Science of the U.S. Department of Energy under Contract No. DE-AC02-05CH11231; STFC DiRAC HPC Facilities, funded by UK BIS National E-infrastructure capital grants; and the UK particle physics grid, supported by the GridPP Collaboration. This work was performed in part under DOE Contract DE-AC02-76SF00515.

Author contributions are listed below.

A.I. Malz: Conceptualization, formal analysis, investigation, methodology, software, visualization, writing original draft; initiated project, led development work. P.J. Marshall: Project administration, software, supervision, writing - original draft; advised on statistics, and project design and management.

J. DeRose: Resources; produced the photometry for the fainter mock catalog.

M.L. Graham: Resources, writing - review \& editing; produced the photometry and PDFs for the brighter mock catalog.

S.J. Schmidt: Resources, writing - review \& editing; produced the PDFs for the fainter mock catalog.

R. Wechsler: Resources; assisted in producing the fainter mock catalog photometry.

Software: jupyter (Kluyver et al. 2016), matplotlib (Hunter 2007), numpy (Walt et al. 2011), pathos (McKerns et al. 2012; McKerns \& Aivazis 2010), qp (Malz \& Marshall 2017), scikit-learn (Pedregosa et al. 2011), scipy (Jones et al. 2001)

\section{REFERENCES}

Amaro, V., Cavuoti, S., Brescia, M., et al. 2016, Proc Int Astron Union, 12, 197

Applegate, D. E., von der Linden, A., Kelly, P. L., et al. 2014, Mon Not R Astron Soc, 439, 48
Bailer-Jones, C. a. L., Andrae, R., Arcay, B., et al. 2013,

A\&A, 559, A74

Benítez, N. 2000, ApJ, 536, 571 
Bonnett, C., Troxel, M. A., Hartley, W., et al. 2016, Phys. Rev. D, 94, 042005

Carrasco Kind, M., \& Brunner, R. J. 2014a, Mon Not R Astron Soc, 438, 3409

-. 2014b, Mon Not R Astron Soc, 441, 3550

Cavuoti, S., Amaro, V., Brescia, M., et al. 2017, Mon Not R Astron Soc, 465, 1959

Choi, A., Heymans, C., Blake, C., et al. 2016, Mon Not R Astron Soc, 463, 3737

Clampitt, J., Sánchez, C., Kwan, J., et al. 2017, Mon Not R Astron Soc, 465, 4204

Connolly, A. J., Angeli, G. Z., Chandrasekharan, S., et al. 2014, in Proc. SPIE, Vol. 9150 (Int. Soc. Opt. Eng.), 14

de Jong, J. T. A., Kleijn, G. A. V., Erben, T., et al. 2017, A\&A, 604, A134

De Vicente, J., Sánchez, E., \& Sevilla-Noarbe, I. 2016, Mon Not R Astron Soc, 459, 3078

Fèvre, O. L., Vettolani, G., Garilli, B., et al. 2005, A\&A, 439, 845

Gonzalez-Perez, V., Lacey, C. G., Baugh, C. M., et al. 2014, Mon Not R Astron Soc, 439, 264

Graham, M. L., Connolly, A. J., Ivezić, Ž., et al. 2018, AJ, 155,1

Harnois-Déraps, J., Tröster, T., Chisari, N. E., et al. 2017, Mon Not R Astron Soc, 471, 1619

Hildebrandt, H., Viola, M., Heymans, C., et al. 2017, Mon Not R Astron Soc, 465, 1454

Hoyle, B., Gruen, D., Bernstein, G. M., et al. 2017, Dark Energy Survey Year 1 Results: Redshift distributions of the weak lensing source galaxies, Tech. Rep. Fermilab PUB-17-293-AE

Hunter, J. D. 2007, Computing in Science Engineering, 9, 90

Ilbert, O., Arnouts, S., McCracken, H. J., et al. 2006, A\&A, 457,841

Ivezić, Ž., Tyson, J. A., Abel, B., et al. 2008

Jones, E., Oliphant, T., \& Peterson, P. 2001, SciPy: Open Source Scientific Tools for Python

Juric, M., Axelrod, T., Becker, A. C., et al. 2017, LSST

Data Products Definition Document
Kluyver, T., Ragan-Kelley, B., Pérez, F., et al. 2016, in ELPUB, 87-90

Laycock, S., Cappallo, R., Williams, B. F., et al. 2017, ApJ, 836,50

LSST Science Collaboration. 2009, ArXiv e-prints, arXiv:0912.0201

Malz, A., \& Marshall, P. 2017, qp, doi:10.5291/zenodo.1133465

McKerns, M., \& Aivazis, M. 2010, pathos: a framework for heterogeneous computing

McKerns, M. M., Strand, L., Sullivan, T., Fang, A., \& Aivazis, M. A. G. 2012, in Proc 10th Python in Science Conf

Merson, A. I., Baugh, C. M., Helly, J. C., et al. 2013, Mon Not R Astron Soc, 429, 556

Pedregosa, F., Varoquaux, G., Gramfort, A., et al. 2011, J Machine Learning Res, 12, 2825

Pizzocaro, D., Stelzer, B., Paladini, R., et al. 2016, A\&A, 587, A36

Polsterer, K. L., D'Isanto, A., \& Gieseke, F. 2016

Radovich, M., Puddu, E., Bellagamba, F., et al. 2017, A\&A, 598, A107

Sadeh, I., Abdalla, F. B., \& Lahav, O. 2016, PASP, 128, 104502

Sheldon, E. S., Cunha, C. E., Mandelbaum, R., Brinkmann, J., \& Weaver, B. A. 2012, ApJS, 201, 32

Springel, V., White, S. D. M., Jenkins, A., et al. 2005, Nature, 435, 629

Sun, W., Grijs, R. d., Fan, Z., \& Cameron, E. 2015, ApJ, 816,9

Tanaka, M., Coupon, J., Hsieh, B.-C., et al. 2018, Publ Astron Soc Jpn Nihon Tenmon Gakkai, 70, doi:10.1093/pasj/psx077

Tröster, T., Camera, S., Fornasa, M., et al. 2017, Mon Not R Astron Soc, 467, 2706

Walt, S. v., Colbert, S. C., \& Varoquaux, G. 2011, Computing in Science \& Engineering, 13, 22 